\title{
Micro management
}

\author{
By Matthew Mikulski, Staff Writer
}

In the past four years, at least two biotechs have released tests that use mRNA-based gene signatures to predict the likelihood of breast cancer recurrence. Now, research is emerging that suggests microRNAs could also have utility in breast cancer prognosis and disease classification. Companies developing miRNA-based tests think their products could have advantages over mRNA-based ones, whereas the mRNA test developers think that miRNA is unlikely to provide any additional prognostic power.

miRNAs are noncoding RNAs that regulate gene expression. Each individual miRNA modulates multiple RNAs. Two papers, in Nature and Nature Cell Biology, detail how five miRNAs function in breast cancer, including three that suppress metastasis and two that promote it.

The Nature paper, from researchers at Memorial Sloan-Kettering Cancer Center, shows that miR-126, miR-206 and miR-335

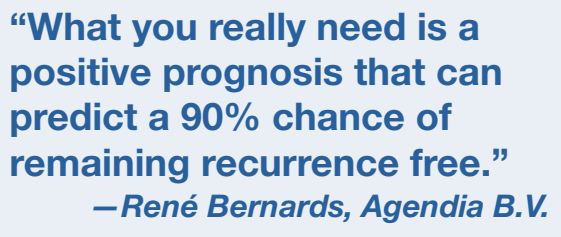

miR-373 was previously identified as oncogenic in testicular germcell tumors. ${ }^{3}$

These two miRNAs were found to downregulate three genes known to slow tumor progression: TFF1, CD44 and BNIP3L. Unlike with miR-335, however, studies looking at breast cancer primary tumor samples suggest that miR-373 on its own cannot sort patients into high- and low-risk groups. The researchers are currently looking at the expression of miR-520c and other family members in breast cancer samples.

Wistar's Qihong Huang, the primary author on the Nature Cell Biology paper, told SciBX that miR-373 should have greater predictive power in the lymph nodes, where tumor cells are more likely to be absent in nonmetastatic patients. Huang said his group is now profiling the lymph node expression of miR-373 and miR-520c, as well as that of miR-520c-related miRNAs, in samples from about 100 patients.

\section{Predicting metastatic disease}

A key implication of the two papers is that the five miRNAs could be used in miRNA-based tests to predict the likelihood of metastatic disease in breast cancer patients. The question is how an miRNA test would stack up against mRNA tests for disease prognosis.

René Bernards, CSO and cofounder of Agendia B.V., said the Nature paper adds to growing evidence that miRNAs are highly potent regulators of cellular processes. However, he also said that neither a combination are underexpressed in metastatic breast cancer cells compared with nonmetastatic cancer cells. In cellular assays, cells with the miRNAs showed less tumor-cell invasion and migration than cancer cells lacking the miRNAs. ${ }^{1}$

Also, data from metastatic breast cancer patient samples shows that only $40 \%$ of patients who lack both miR-126 and miR-335 are likely to be metastasis free at 5 years, compared with about $80 \%$ of patients who express the miRNAs. Joan Massagué, who headed the group that conducted the research, could not be reached to comment on why the prognostic potential of the third miRNA was not examined.

Of the three miRNAs, the Sloan-Kettering group said miR-335 was the strongest predictor, and they identified six genes it regulates: COL1A1, PTPRN2, MERTK, PLCB1, tenascin C (TNC) and SOX4. The genes code for proteins involved in cytoskeletal control, signal transduction, cell migration and transcriptional control.

When applied to 368 samples from breast cancer patients, the sixgene signature sorted patients into a higher risk category, defined as about a $50 \%$ chance of metastasis at 10 years, and a lower risk category, defined as about a $30 \%$ chance.

Although the three miRNAs in the Nature paper probably have a role in suppressing metastasis, the paper in Nature Cell Biology by researchers at The Wistar Institute and The Netherlands Cancer Institute shows that overexpression of two other miRNAs-miR-373 and miR-520c-had a prometastatic effect in breast cancer cells. ${ }^{2}$ of the miRNAs examined nor the six-gene signature derived from miR335 has sufficient predictive power. Bernards declined to comment on the Nature Cell Biology paper.

"What you really need is a positive prognosis that can predict a $90 \%$ chance of remaining recurrence free," he said. According to Bernards, this level of differentiation provides the physician with a sufficient difference in expected outcome to support therapy decisions.

Agendia launched its MammaPrint mRNA-based breast cancer prognostic test in the U.S. in December 2007. The test, which consists of a 70-gene signature, is used to predict the likelihood of breast cancer recurrence within 5-10 years. Patients identified by the test as low risk have a $90 \%$ chance of remaining recurrence free at 10 years.

Bernards noted that MammaPrint and other prognostic gene-signature tests for breast cancer recurrence, including Oncotype DX from Genomic Health Inc., use different gene panels but generally give similar results. Because of this, he said, "I would predict that miR-335 or its gene signature wouldn't add much."

He did tell SciBX that an miRNA-based test could have commercial applicability if enough miRNAs with additive predictive power were identified and combined.

Ranit Aharonov, EVP of diagnostics at miRNA company Rosetta Genomics Ltd., argued that an all-miRNA test could deliver more consistent and potentially more powerful results than an mRNA-based test. 
Rosetta plans to launch its first miRNA-based tests this year. These include a diagnostic based on a single, undisclosed miRNA to help pathologists differentiate between squamous cell and non-squamous cell lung cancers, and another diagnostic incorporating a panel of about 52 miRNAs for determining the originating cancer type in patients with cancer of unknown primary origin.

A third project at Rosetta-a test to aid in the prognosis of mesothelioma-is based on a panel of four undisclosed miRNAs. A timeline for the product has not been disclosed.

According to Aharonov, recent research suggests that miRNAs are more reliable descriptors of cancer cell type and activity than mRNAs because the former are more stable and because individual miRNAs regulate multiple genes. ${ }^{4}$ The increased predictive power, she said, would come from the fact that each miRNA regulates a bundle of genes.

Aharonov added that mRNAs cannot be reliably isolated from blood because the molecules are rapidly degraded.

\section{miRNA therapy}

Because all five miRNAs described in Nature and Nature Cell Biology were demonstrated not only to be biomarkers but also to produce measurable cancer phenotypes in vitro and to affect disease outcomes in vivo, their regulatory functions might also be used to therapeutic ends.

The prometastatic miR-373 and miR-520c could be knocked down by an antisense molecule incorporating a complementary nucleotide sequence. Developing miRNA mimics for the three tumor-suppressing miRNAs could be a more challenging task.

"We've elected to focus on antagonists because we think they would be less problematic," said Henrik Oerum, VP and CSO at RNA therapeutics company Santaris Pharma A/S.

Santaris is developing mRNA and miRNA antagonists based on its locked nucleic acid technology. In December 2007, the company partnered with GlaxoSmithKline plc to develop RNA antagonists as antiviral therapies. Santaris also gave the pharma company an option to take over development of SPC3649 once it completes Phase IIa testing. The antagonist of miR-122 is in preclinical development to treat $\mathrm{HCV}$, with Phase I testing expected to start in $1 \mathrm{H} 08$.

miR-122 is present in HCV and is used by it to facilitate replication. ${ }^{5}$

There are two main challenges to replicating an miRNA's activity: ensuring that the mimic gets into the right cells and that, once there, it efficiently integrates into the RNA-induced silencing complex (RISC).

Because miRNAs are highly cell specific, miRNA-mimicking therapeutics that get into nontarget cells could potentially create undesirable gains of function. According to Oerum, this may be more risky than the loss of function an miRNA antagonist might cause.

Even if miRNA mimics could be delivered to the intended cells, Oerum said, uptake and stability still would be an issue. The reason is that the RISC naturally processes double-stranded transcripts to produce miRNAs, which are much larger and more electrostatic than the single strands needed for miRNA antagonism. The double-stranded transcripts are around " 13,000 daltons, compared to 6,000-7,000 for standard antisense and 3,000-4,000 for an optimized miRNA knockdown agent," Oerum said.

Kleanthis Xanthopoulos, president and CEO of Regulus Therapeutics LLC, agreed that there are several hurdles to creating miRNA mimics for therapeutic use. Regulus is developing new chemistries that provide for interactions with the RISC without having to deliver a double-stranded molecule.

He also noted that new strategies are being developed to more precisely deliver mimics. Examples, said Xanthopoulos, include "receptor-mediated introduction of the molecules or cholesterol-conjugated molecules affinitive for certain tissues."

Regulus, a 50/50 joint venture between short interfering RNA company Alnylam Pharmaceuticals Inc. and RNA therapeutics company Isis Pharmaceuticals Inc., is focusing specifically on miRNA-based therapeutics. The company's lead program is an miR-122 antagonist in preclinical development to lower triglyceride levels, prevent fatty liver and treat HCV. The plan is to be in the clinic with one of the indications by $1 \mathrm{H} 09$.

The authors of the Nature paper have submitted a patent application claiming use of the miR-335 signature, miR-126 and miR-335 as diagnostics, as well as the therapeutic use of the miRNAs. Wistar has submitted a patent application around the second paper covering the diagnostic and therapeutic use of miR-373 and miR-520c.

\section{REFERENCES}

1. Tavazoie, S. et al. Nature; published online Jan. 9, 2008; doi:10.1038/ nature 06487

Contact: Joan Massagué, Memorial Sloan-Kettering Cancer Center, New York, N.Y. e-mail: massaguj@mskcc.org

2. Huang, Q. et al. Nat. Cell Biol.; published online Jan. 13, 2008; doi:10.1038/ncb1681

Contact: Reuven Agami, The Netherlands Cancer Institute, Amsterdam, The Netherlands

e-mail: r.agami@nki.nl

Contact: Qihong Huang, The Wistar Institute, Philadelphia, Pa. e-mail: qhuang@wistar.org

3. Voorhoeve, P. et al. Cell 124, 1169-1181 (2006)

4. Lu, J. et al. Nature 435, 834-838 (2005)

5. Jopling, C. et al. Science 309, 1577-1581 (2005)

\section{COMPANIES AND INSTITUTIONS MENTIONED}

Agendia B.V., Amsterdam, The Netherlands

Alnylam Pharmaceuticals Inc. (NASDAQ:ALNY), Cambridge, Mass. Genomic Health Inc. (NASDAQ:GHDX), Redwood City, Calif. GlaxoSmithKline plc (LSE:GSK; NYSE:GSK), London, U.K. Isis Pharmaceuticals Inc. (NASDAQ:ISIS), Carlsbad, Calif. Memorial Sloan-Kettering Cancer Center, New York, N.Y. The Netherlands Cancer Institute, Amsterdam, The Netherlands Regulus Therapeutics LLC, Carlsbad, Calif.

Rosetta Genomics Ltd. (NASDAQ:ROSG), Rehovot, Israel

Santaris Pharma A/S, Horsholm, Denmark The Wistar Institute, Philadelphia, Pa. 